\title{
Chinese EFL Learners' Acquisition of English Relative Clauses
}

\author{
Qing-Qiang Gao ${ }^{1}$ \\ ${ }^{1}$ College of Humanities and Foreign Languages, Xi' an University of Science and Technology, China \\ Correspondence: Qing-Qiang Gao, College of Humanities and Foreign Languages, Xi'an University of Science \\ and Technology, Lintong, Xi’an 710600, Shaanxi, China. E-mail:gaoqq69@yahoo.com
}

Received: March 10, 2014 Accepted: April 11, 2014 Online Published: May 27, 2014

doi:10.5539/ijel.v4n3p82

URL: http://dx.doi.org/10.5539/ijel.v4n3p82

\begin{abstract}
The study attempts to investigate Chinese English learners' difficulty in the acquisition of English relative clauses (RCs). In addressing the objective, the present researcher employed two tests, sentence combination test and grammaticality judgment test, which were borrowed from Izumi (2003), to examine the learners' knowledge of English RCs. 40 subjects participated in the study. In the sentence combination test, the subjects were instructed to combine two sentences together in a way so that relative clause would be formed. In the grammaticality judgment test, the subjects were supposed to judge the grammaticality of the given sentences and provide corrections for those they regard ungrammatical.

Data obtained from the two tests reveal that RCs modifying object are much easier than those modifying subject. The study found full support for the Kuno's (1974) PDH, which proposes that center-embedding is more difficult to acquire than right-embedding due to short term memory limitations. The other two hypotheses, Keenan and Comrie's (1977) Noun Phrase Accessibility Hierarchy Hypothesis (NPAH), and Hamilton's (1994) Subject-Object Hierarchy Hypothesis (SOHH) were not borne out.
\end{abstract}

Keywords: English relative clauses, the NPAH, the SOHH, the PDH

\section{Introduction}

English relative clause is a type of complex post nominal adjectival modifier used in both written and spoken English. It is a type of embedded clause, which is noun modification construction in which one clause is subordinate to another.

So far, the acquisition of relative clauses (RCs) has played an important role in both linguistic and psycholinguistic study. The issue has been studied extensively by many a researcher studying both first language acquisition and second language acquisition.

A lot of hypotheses have been put forward to account for the acquisition of English relative clauses. And some were meant to illustrate the relative ease and difficulty in acquiring different types of RC sentences and among them, three hypotheses have received most attention, namely Keenan and Comrie's (1977) Noun Phrase Accessibility Hierarchy Hypothesis (NPAH), Kuno's (1974) Perceptual Difficulty Hypothesis (PDH) and Hamilton's (1994) Subject-Object Hierarchy Hypothesis (SOHH). Many studies have been carried out to check these three hypotheses in both first language and second language. But it was the researchers abroad that conducted most of those studies. Meanwhile, those studies are different in the focus of investigation, methodology and participants. In addition, so far very few empirical studies have been conducted to examine the Chinese English learners' processing difficulty of English relative clauses in Chinese context. Thus such a study will enable researchers to find useful insights in understanding the Chinese English learners' acquisition of RCs as well as the process of second language acquisition.

\section{Literature Review}

\subsection{Noun Phrase Accessibility Hierarchy (NPAH)}

Regarding the acquisition orders of the different types of RCs, most studies refer to Keenan and Comrie's (1977) Noun Phrase Accessibility Hierarchy (NPAH). After a detailed comparative study of RC structures in different languages, Keenan and Comrie proposed the NPAH, which suggests the following universal order of from easiest to most difficult, as shown in (1).

(1) SU $>$ DO $>$ IO $>$ PREP $>$ GEN $>$ COMP 
The symbol $>$ here denotes "is more accessible than".

Table 1 shows example sentences of RCs formed on each position in the hierarchy. According to the NPAH, if a language can form RCs on a given position in the hierarchy, it can also form RCs on all positions higher (or to the left) in the hierarchy, but the reverse is not true. For example, if a language can form RCs on OPREP, it can also form RCs on SU, DO, and IO, but not necessarily GEN or OCOMP. The NPAH reflects the natural order of acquisition and it predicts that RCs formed on the subject are easiest to learn, while those on the object of a comparative are the most difficult.

Table 1. Example sentences for different RC types in the NPAH

\begin{tabular}{cc}
\hline RC type & Example \\
\hline SU (Subject) & the girl that came... \\
DO (Direct Object) & the girl that John hit... \\
IO (Indirect Object) & the girl that he spoke to... \\
OPREP (Object of Preposition) & the girl that he sat near... \\
GEN (Genitive) & the girl whose father died... \\
OCOMP (Object of Comparative) & the girl that he is taller than... \\
\hline
\end{tabular}

\subsection{Perceptual Difficulty Hypothesis (PDH)}

Another influential hypothesis regarding the acquisition order of different types of RCs is Kuno's (1974) Perceptual Difficulty Hypothesis (PDH). Based on the limitation of the human memory system, the PDH predicts that due to short term memory limitations, center embedding is perceptually more difficult to process than right embedding, because center embedding interrupts the processing of the matrix sentence while right embedding doesn't. Thus the sentence in (2) is claimed to be more difficult to process than sentence in (3).

(2) center embedding

The boy that the woman that the man loved scolded was clever.

(3) right embedding

The man loved the woman that scolded the boy that was clever.

With regard to RCs, the PDH predicts that RCs formed on the subject are more difficult than those formed on the object. To be more specific, object embedded, subject focus" (OS) and "object embedded, object focus" (OO) types of RCs should be easier than "subject embedded, subject focus" (SS) and "subject embedded, object focus" (SO) types, as shown in (4).

(4) $\mathrm{OS} / \mathrm{OO}>\mathrm{SS} / \mathrm{SO}$

Note. ">" means "is easier than and/means "as difficult as"

\subsection{Subject-Object Hierarchy Hypothesis (SOHH)}

The third hypothesis that has received certain attention is Hamilton's (1994) SOHH. The SOHH, based on the notion of processing discontinuity, is a composite of the NPAH and the PDH. Processing discontinuity can be formed under two situations. When a relative clause interrupts the main clause, one type of discontinuity will be created. When the RC is placed between the relative pronoun and the wh-trace created by relativization, the other type of discontinuity will emerge. This means that embedded object wh-traces created by relativization set up two phrasal discontinuities within the RC, whereas embedded subject wh-traces set up only a single discontinuity, as illustrated in Table 2 (from Hamilton, 1994, p. 135).

Table 2. Discontinuities in SS and SO types of RC

$\begin{array}{ll}\text { relativized subject: } & \text { The man who }{ }_{i}\left[t_{i} s a w ~ u s\right] \\ \text { relativized direct object: } & \text { The man who }{ }_{i}\left[s w e\left[v p s a w t_{i}\right]\right.\end{array}$

Note. [ ] $=$ phrasal boundary; $\mathrm{t}=$ wh-trace; $\mathrm{i}=$ co-index; $\mathrm{S}=$ sentential node; $\mathrm{VP}=$ verb phrase. 
The SOHH proposes an implicational relationship between four types of RC clauses, namely, OS, OO, SS and SO. For each label the first code refers to the head noun as either subject (S) or direct object (O) of the matrix clause, and the second code refers to the role of the NP target of relativization within the relative clause.

The SOHH predicts the following difficulty order, as is shown in (5).

(5) $\mathrm{OS}>\mathrm{OO} / \mathrm{SS}>\mathrm{SO}$

Note. ">" means "is easier than and/means "as difficult as"

According to the SOHH, the number of discontinuities in the structure determines the difficulty. Thus, OS types should be the easiest to acquire because they contain only one discontinuity within the RC. With two discontinuities OO types are more difficult than OS. Similarly the SS types contain two discontinuities and are of equal difficulty to the $\mathrm{OO}$ types. With three discontinuities the SO types are presumed to be the most difficult to acquire.

The most recent study of English relative clause acquisition was conducted by Izumi (2003). The subjects' L1s included Arabic (24), Chinese (6), French (2), Japanese (3), Kazah (1), Korean (11), Persian (1), Portuguese (1), Spanish (6), Thai (4) and Turkish (1). Testing instruments were sentence combination test, picture-cued sentence interpretation test and grammaticality judgment test. Izumi's study found mixed support for the NPAH and the SOHH and full support for the PDH.

Izumi's study is one of the most important studies investigating the knowledge of EFL learners on English RCs. He used multiple measures to test learners' interlanguage of the target structure. Furthermore, his study gives both methodological and theoretical insights for future research.

To sum up, the research on the acquisition of English RC s has a long history. Ample studies have been conducted and different accounts have been put forward from different perspectives. However, no consistent conclusions have been reached due to different research focus, different methodologies as well as different rationales.

\section{Methodology}

\subsection{Research Questions}

As is mentioned earlier, the present study aims to investigate Chinese EFL learners' acquisition of English relative clauses by testing three hypotheses regarding RC acquisition. Specifically, it attempts to address the following research questions:

1. Which hypothesis best predicts Chinese EFL learners' difficulty in the acquisition of English RCs, the NPAH, the $\mathrm{PDH}$ or the SOHH?

2. In what way are the results of the present study different from those of the prior study and what are the possible reasons for the differences?

\subsection{Subjects}

40 sophomore English majors from Xi'an Translators' College participated in the experiment. Among the 40 participants, 6 were male while 34 were female. Their mean age was 20. They had similar English learning experience and they had learned English as a foreign language for 7 years. They started to learn English in the junior middle school in a formal classroom setting, 5 hours a week. All of them passed a stringent and highly competitive national exam of English before they were admitted in the English department of the college.

Before the tests, the participants were informed that the results of the tests would be calculated as one part of their grades in the final examination. Thus all the participants were well motivated in the experiment.

\subsection{Research Instrument}

Since this is a replication study of Isumi's (2003), the instrument employed by the present researcher was borrowed from Isumi (2003), who borrowed from Doughty (1991) and Gass (1982), and modified it. Following Isumi (2003), two testing instruments were employed to examine the participants' knowledge of RCs, a sentence combination test and a grammaticality judgment test. The former was used to examine the participants' productive ability while the latter was meant to examine their intuitional knowledge. This study concentrated on only three of the six types of RCs investigated in the NPAH: SU, DO and OPREP. Half of the targeted RCs were subject-modifying and half were object-modifying.

The task of carrying out the tests was entrusted to the teacher of the class. The tests were conducted in the procedure as specified by the present researcher. The sentence combination test was administered first, followed 
by the grammaticality judgment test. Directions for the tests were provided in Chinese so that all subjects would have a clear understanding of the purpose of the tests and how to complete them. In each test the experimenter explained relevant example before the participants started the test so as to make sure that the participants were clear about how to complete the tests as required. The participants were required to finish the tests in limited time and they were not allowed to make changes after they finished the test. This would guarantee that they respond intuitively.

\subsection{Scoring}

The scoring method followed the procedure adopted in Izumi's (2003) study. The six types of English RCs were scored separately.

For the sentence combination test, 1 point would be scored only when the intended RCs were produced and 0 point would be for those unintended RCs. Specifically, if the subject produced an SS RC for an item for which an SO RC was expected, the answer would be considered incorrect. Such errors as spelling, tense were neglected on condition that they were not relevant to the production of the RC.

The grammaticality judgment test was scored with great care. The participants' corrections of errors were examined first and this was to guarantee the participants judged the tested item based on the expected form. If the participants' correction for a specific item revealed that the sentence was judged as ungrammatical for reasons irrelevant to the RC-related problems, that item would be inapplicable and was rejected. Thus it's necessary to reject a few test items if the participants failed to accomplish the task timely or follow the process as required.

RC-related corrections typically involved movement of the RC to an adjacent position of the head noun if it was not already there, change of the word order inside the $\mathrm{RC}$, change of the relative pronoun, insertion of a relative pronoun when there was none. Non-RC-related corrections included tense and aspect change and change of prepositions in the matrix clause.

Items without judgment or corrections were dismissed from the analysis. As a result, the number of total items for each participant varied from each other and through dividing the total correct scores by the total number of applicable items, the scores for each participant could be worked out. Therefore, each participant got a percentage score for each type of $\mathrm{RC}$ in both the two tests. 1 point was scored for the correct judgment and 0 for those incorrect. For example, if a participant produced all the SO type of RCs in the sentence combination test, the score for the SO would be $100 \%$. If two were produced, the score would be $66.7 \%$. The grammaticality judgment was much more complex. If a participant made correct judgment for all the SO type, the score would be $100 \%$. If only two were judged correctly and one was left unjudged, the score was still $100 \%$ since the unjudged one was inapplicable to the analysis and thus excluded.

\section{Results and Discussion}

\subsection{Results of the Sentence Combination Test}

The mean accuracy scores for each type of RCs placed in the two different matrix positions are presented in Table 3. The aggregate results for matrix subject position versus matrix object position are displayed in the rightmost column.

Table 3. Descriptive statistics of sentence combination test

\begin{tabular}{lllll}
\hline \multirow{4}{*}{ Matrix position } & SU & DO & OPREP & Total \\
\hline Subject & $55.8(44.3)$ & $55.8(38.8)$ & $38.3(37.4)$ & $50.0(40.8)$ \\
Object & $75.8(31.1)$ & $75.8(31.1)$ & $62.5(37.9)$ & $71.4(31.8)$ \\
\hline
\end{tabular}

Note. Standard deviations are given in parentheses.

To investigate statistical significance of the observed differences, we conducted a two-way ANOVA, repeated measures using a 2 (matrix subject versus matrix object) x 3 (RC Types: SU versus DO versus OPREP). The Repeated Measures ANOVA revealed significant main effects for the within-subjects factor: RC type ( $F$ $(2,117)=3.6, p=.030)$, and for the between-subjects: matrix positions $(F(1,117)=27.908, p<.0001)$, but no interaction effect for $\mathrm{RC}$ type by matrix position $(\mathrm{F}=(2, .117), \mathrm{p}=.889)$. 
A post hoc analysis was carried out to investigate the main effect for the RC type, and the test found no effect for any comparisons on either matrix subject and object position. Pairwise Comparisons found significant effect only for the comparison between OS and OPRE ( $\mathrm{p}=.022)$.

As is revealed from Table 3 that concerning RC types, SU and DO obtained equal score, which is higher than OPREP.

With regards to the matrix positions, the subject position obtained a lower score than the object position, which is consistent with the order predicted by the PDH. RCs modifying the object are much easier than those modifying the subject. And a difficulty order can be derived from Table 3 as follows: $\mathrm{OO} \geq \mathrm{OS}>\mathrm{OOPrep}>\mathrm{SO}$ $\geq$ SS > SOPrep. Interestingly, it can be observed that RC types embedded in the matrix object position fall into the first three, whereas those embedded in the matrix subject position fall into the rest.

\subsection{Results of the Grammaticality Judgement Test}

Table 4. Descriptive statistics of grammaticality judgement test

\begin{tabular}{lllll}
\hline \multirow{4}{*}{ Matrix position } & SU & DO & OPREP & Total \\
\hline Subject & $71.3(21.5)$ & $64.2(26.6)$ & $65.5(26.8)$ & $67.0(25.1)$ \\
Object & $89.1(14.8)$ & $77.4(21.2)$ & $88.4(24.7)$ & $84.9(21.2)$ \\
\hline
\end{tabular}

Note. Provided in parentheses are standard deviations.

Table 4 displays the results of the accuracy scores for each RC sentence type on the grammaticality judgment test.

To check the statistical significance of the observed differences, the test scores were subjected to a two-way ANOVA, repeated measures using a 2 (matrix subject versus matrix object positions) x 3 (RC Types: SU versus DO versus OPREP). The two-way repeated-measures ANOVA revealed no significant main effect for the within-subject factor: $R C$ types $(F(2,117)=2.73, p=.069)$ and no significant interaction effect $(F(2,117)=1.205$, $\mathrm{p}=.303$ ). Pairwise Comparisons of the three RC types showed significant effect only for the pair between SU and DO $(\mathrm{p}=.023)$.

However, Tests of between-subjects found main effects for the matrix position, $F(1,117)=49.27, p<.0001$. Multiple Comparisons of Post Hoc Scheffe found no effect for matrix subject position. The Only effect was found between $\mathrm{SU}$ and DO ( $\mathrm{p}=.043)$ in the Object position, as shown in Table 5.

As is revealed from Table 4, with regards to RC types, SU obtained the highest score, followed by OPREP, and then DO, which is not consistent with the NPAH. Concerning the matrix position, the higher score was obtained by the object position rather than the subject position, which is exactly the order predicted by the PDH. The difficulty order can be obtained from the grammaticality judgment test as follows: OS $>$ OOPrep $>\mathrm{OO}>\mathrm{SS}>$ SOPrep $>$ SO. What is consistent with the results of the sentence combination test is that RC types embedded in the matrix object position occupy the first three, whereas those embedded in the matrix subject position occupy the last three. 
Table 5. Post hoc multiple comparisons of the grammaticality judgment test

\begin{tabular}{lllll}
\hline Dependent Variable & (I) RCTYPES & (J) RCTYPES & Mean Difference (I-J) & Sig. \\
\hline SUBJECT & SS & SO & 7.0050 & .462 \\
& & OPS & 5.7450 & .594 \\
& SO & SS & -7.0050 & .462 \\
& & OPS & -1.2600 & .975 \\
OBJECT & OPS & SS & -5.7450 & .594 \\
& OS & OO & 1.2600 & .975 \\
& & OPO & 11.7525 & .043 \\
& OO & OS & -11.7525 & .987 \\
& & OPO & -11.0040 & .043 \\
& OPO & OS & -.7485 & .063 \\
& & OO & 11.0040 & .063
\end{tabular}

* The mean difference is significant at the .05 level.

\section{Conclusion}

The difficulty order obtained from the sentence combination test is $\mathrm{OO} \geq \mathrm{OS}>\mathrm{OOPrep}>\mathrm{SO} \geq \mathrm{SS}>\mathrm{SOPrep}$ and that of the grammaticality judgment test is OS $>$ OOPrep $>$ OO $>$ SS $>$ SOPrep $>$ SO. Results from both the sentence combination test and the grammaticality judgment test show that RCs modifying the object are much easier than those modifying the subject. This is exactly the order predicted by the PDH. Neither the NPAH nor the SOHH can be verified by the present study.

Data obtained from two tests reveal that object-modifying RCs are much easier than the subject-modifying RCs. The study found full support for the Kuno's (1974) PDH, which predicts that center-embedding is more difficult to process than right-embedding due to short term memory limitations. The other two hypotheses, Keenan and Comrie's (1977) Noun Phrase Accessibility Hierarchy Hypothesis (NPAH), and Hamilton's (1994) Subject-Object Hierarchy Hypothesis (SOHH) were not borne out. It is suggested that the PDH offers a plausible account for the difficulty in the EFL learners' acquisition of English relative clauses.

\section{References}

Doughty, C. (1991). Second language instruction does make a difference. Studies in Second Language Acquisition, 13, 431-469. http://dx.doi.org/10.1017/S0272263100010287

Gass, S. (1982). From theory to practice. In M. Hynes, \& W. Rutherford (Eds.), On TESOL' 81: Selected Papers from the Fifteenth Annual Conference of Teachers of English to Speakers of Other Languages (pp. 129-139). Washington, DC: TESOL.

Hamilton, R. L. (1994). Is implicational generalization unidirectional and maximal? Evidence from relativization instruction in a second language. Language Learning, 44, 123-157. http://dx.doi.org/10.1111/j.1467-1770.1994.tb01096.x

Izumi, S. (2002). Processing difficulty in comprehension and production of relative clauses by learners of English as a second language. Language Learning, 53(2), 285-323. http://dx.doi.org/10.1111/1467-9922.00218

Keenan, E., \& Comrie, B. (1977). Noun phrase accessibility and universal grammar. Linguistic Inquiry, 8, 63-99.

Kuno, S. (1974). The position of relative clauses and conjunctions. Linguistic Inquiry, 5, 117-136.

\section{Copyrights}

Copyright for this article is retained by the author(s), with first publication rights granted to the journal.

This is an open-access article distributed under the terms and conditions of the Creative Commons Attribution license (http://creativecommons.org/licenses/by/3.0/). 